Author affiliations and support information (if applicable) appear at the end of this article.

Published at jco.org on March 28, 2017. Clinical trial information: NCT01015352, NCT01718379

Corresponding author: Sophie Park, $\mathrm{MD}, \mathrm{PhD}$, Clinique Universitaire d'Hématologie, $\mathrm{CHU}$ de Grenoble Alpes, CS 10217, 38043 Grenoble Cedex 09, France; e-mail: spark@chu-grenoble.fr.

(c) 2017 by American Society of Clinical Oncology

$0732-183 X / 17 / 3514 w-1591 w / \$ 20.00$

\title{
Outcome of Lower-Risk Patients With Myelodysplastic Syndromes Without $5 q$ Deletion After Failure of Erythropoiesis-Stimulating Agents
}

Sophie Park, Jean-François Hamel, Andrea Toma, Charikleia Kelaidi, Sylvain Thépot, Maria Diez Campelo, Valeria Santini, Mikkael A. Sekeres, Enrico Balleari, Jennifer Kaivers, Rosa Sapena, Katharina Götze, Catharina Müller-Thomas, Odile Beyne-Rauzy, Aspasia Stamatoullas, Ioannis Kotsianidis, Rami Komrokji, David P. Steensma, Jaime Fensterl, Gail J. Roboz, Teresa Bernal, Fernando Ramos, Marisa Calabuig, Agnès Guerci-Bresler, Dominique Bordessoule, Pascale Cony-Makhoul, Stéphane Cheze, Eric Wattel, Christian Rose, Norbert Vey, Daniela Gioia, Dario Ferrero, Gianluca Gaidano, Giovanni Cametti, Fabrizio Pane, Alessandro Sanna, Ulrich Germing, Guillermo F. Sanz, François Dreyfus, and Pierre Fenaux

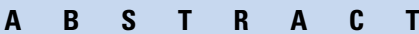

\section{Purpose}

Most anemic patients with non-deleted $5 q$ lower-risk myelodysplastic syndromes (MDS) are treated with erythropoiesis-stimulating agents (ESAs), with a response rate of approximately $50 \%$. Secondline treatments, including hypomethylating agents (HMAs), lenalidomide (LEN), and investigational drugs, may be used after ESA failure in some countries, but their effect on disease progression and overall survival (OS) is unknown. Here, we analyzed outcome after ESA failure and the effect of second-line treatments.

\section{Patients and Methods}

We examined an international retrospective cohort of 1,698 patients with non-del(5q) lower-risk MDS treated with ESAs.

\section{Results}

Erythroid response to ESAs was 61.5\%, and median response duration was 17 months. Of 1,147 patients experiencing ESA failure, 653 experienced primary failure and 494 experienced relapse after a response. Primary failure of ESAs was associated with a higher risk of acute myeloid leukemia $(A M L)$ progression, which did not translate into an OS difference. Of 450 patients (39\%) who received second-line treatment, 194 received HMAs, 148 received LEN, and 108 received other treatments (MISC), whereas 697 received RBC transfusions only. Five-year AML cumulative incidence was $20.3 \%, 20.3 \%$, and $11.3 \%$ for those receiving HMAs, LEN, and MISC, respectively $(P=.05)$. Five-year OS for patients receiving HMA, LEN, and MISC was $36.5 \%, 41.7 \%$, and $51 \%$, respectively $(P=.21)$. In a multivariable analysis adjusted for age, sex, revised International Prognostic Scoring System score, and progression at ESA failure, there was no significant OS difference among the three groups.

\section{Conclusion}

In this large, multicenter, retrospective cohort of patients with non-del(5q) lower-risk MDS treated with ESAs, none of the most commonly used second-line treatments (HMA and LEN) significantly improved OS. Early failure of ESAs was associated with a higher risk of AML progression.

\section{J Clin Oncol 35:1591-1597. (C) 2017 by American Society of Clinical Oncology}

\section{INTRODUCTION}

Myelodysplastic syndromes (MDS) are clonal diseases of hematopoietic cells occurring in older patients, characterized by ineffective hematopoiesis and anemia in a majority of those affected. Disease evolution is typified by worsening cytopenias and acute myeloid leukemia (AML) transformation. ${ }^{1}$

Erythropoiesis-stimulating agents (ESAs) are often the first line of treatment for anemia in patients with lower-risk MDS who lack the $5 q$ deletion $[\operatorname{del}(5 q)]$. Response rates range from $30 \%$ to $60 \%$, with a median duration of response of 20 to 24 months. $^{2-4}$ Prognostic factors for 
better response to ESAs include low $\mathrm{RBC}$ transfusion requirement, low serum erythropoietin (EPO) level, and lower-risk disease per the revised International Prognostic Scoring System (IPSS-R). ${ }^{5}$ Treatment with ESAs may also be associated with improved survival compared with RBC transfusions alone. ${ }^{2,3}$ However, most responders will experience relapse and become $\mathrm{RBC}$ transfusion dependent. ${ }^{6,7}$

In a previous Groupe Francophone des Myelodysplasies (GFM) study involving 253 patients with lower-risk MDS experiencing ESA failure, we found that AML-free survival and overall survival (OS) were poorer in patients experiencing early ESA failure (ie, with absence of erythroid response or relapse $<6$ months) compared with patients experiencing later relapse after a response. ${ }^{7}$ However, few patients in that cohort had received treatment other than RBC transfusions after ESA failure. Recently, the hypomethylating agents (HMAs) azacitidine (AZA) and decitabine (DAC) and the immunomodulating agent lenalidomide (LEN) demonstrated efficacy in treating anemia in patients with lower-risk MDS without del(5q) and ESA resistance, with RBC transfusion independence rates of $20 \%$ to $40 \%$. $^{8,9}$ These drugs are now relatively widely used for this indication (albeit off label in all countries in the case of LEN and in most countries outside the United States in the case of AZA and DAC). No studies have compared the effect of these drugs on disease progression and OS versus best supportive care (mainly including RBC transfusions). Although other drugs are being tested in patients with lower-risk MDS without del $(5 q)$ after ESA failure, limited numbers of patients have been treated, with short follow-up.

In this study, we analyzed data from the French and Belgian (GFM), Italian (Fondazione Italiana Sindromi Mielodisplastiche [FISM]), Spanish (Grupo Español de Síndromes Mielodisplásicos [GESMD]) cooperative groups, the German registry (Düsseldorf and Münich), and US MDS Clinical Research Consortium cohorts and data from two Greek centers to assess outcome after ESA failure, evaluating in particular the potential effects of primary and secondary failure on outcome and of second-line treatments, compared with best supportive care, on AML progression and OS.

\section{PATIENTS AND METHODS}

\section{Patients}

For our study, patients with a low or intermediate-I IPSS score and non-del(5q) MDS treated with ESAs between 1997 and 2014, for whom ESA treatment failed (primary or secondary failure), without AML or higher-risk MDS progression at the time of ESA failure were eligible. Overall, 1,875 patients with lower-risk MDS according to IPSS were treated with ESAs. We excluded 177 patients who experienced progression to an intermediate-II or high IPSS score at ESA failure to avoid the bias of having more patients at IPSS intermediate-II or high risk in the group treated with HMAs at second-line treatment.

Of the remaining 1,698 patients included, 715 originated from the GFM, 387 from the FISM, and 289 from the GESMD registries, 243 from the US MDS Clinical Research Consortium, and 38 from the German and 26 from the Greek registries; 94 patients had been included in a prospective GFM trial randomly assigning participants to LEN versus LEN combined with EPO, and 76 patients had been included in a GFM trial randomly assigning participants to AZA versus AZA combined with EPO; both trials involved $\mathrm{RBC}$ transfusion-dependent lower-risk MDS patients refractory to ESAs. ${ }^{8,9}$ All patients were classified (or reclassified) per WHO 2008 criteria. Patients with chronic myelomonocytic leukemia, other MDS or myeloproliferative neoplasms, or $\operatorname{del}(5 q)$ MDS were excluded. MDS with myelofibrosis, MDS unclassified, and refractory cytopenia with unilineage dysplasia were classified as MDS unclassified. Refractory anemia (RA) with ring sideroblasts, refractory cytopenia with multilineage dysplasia and ringed sideroblasts, and RA with ring sideroblasts and thrombocytosis were classified as MDS with ring sideroblasts; RA and refractory cytopenia with multilineage dysplasia were combined in the RA without excess blasts subtype.

IPSS-R score was reevaluated in all patients at ESA initiation and ESA failure. All patients received EPO alfa, EPO beta, or darbepoietin for at least 8 weeks.

\section{Definition of ESA Failure}

Primary failure was defined as the absence of erythroid response according to International Working Group 2006 criteria (HI-E) after 8 weeks of ESA treatment. For the 235 patients who received granulocyte colony-stimulating factor after ESA primary failure, ESA response was evaluated after an additional 8 to 12 weeks of treatment with ESAs plus granulocyte colony-stimulating factor treatment. Secondary failures of ESA treatment were defined as relapse after an initial HI-E.

For subsequent follow-up, patients were divided into those who received HMAs, LEN, or other treatments or RBC transfusions only (MISC group). Our combining of patients who received neither HMAs nor LEN into one group (MISC) is justified under Statistical Methods. Second-, third-, and fourth-line treatments were defined as the second, third, and fourth lines of treatment administered after ESA failure, respectively.

\section{Statistical Methods}

Categorical data were described using percentages and compared using Fisher's exact test when appropriate, and continuous data were described using medians and interquartile ranges and compared using the Kruskal-Wallis exact test.

The primary end point was OS after ESA failure, calculated from the date of relapse in patients experiencing secondary ESA failure or from the primary failure assessment conducted 12 weeks after initiation of ESAs for patients with primary resistance. The secondary end point was 5-year cumulative incidence of AML transformation after ESA failure.

Covariates potentially influencing OS or AML cumulative incidence were predefined and included second-line treatment (categorized as MISC, HMAs, or LEN), age (dichotomized as $\leq 75 v>75$ years), sex, IPSS-R score at ESA failure, IPSS-R progression between ESA initiation and ESA failure, and duration of ESA response (dichotomized as $\leq 6 v>6$ months as in the previous GFM study). ${ }^{7,10-12}$

Analysis of the impact of two different second-line treatments (HMAs and LEN) compared with best supportive care and MISC was conducted by considering such second-line treatments as time-dependent covariates, because their effect could only be observed from the moment they were introduced. One of the main study objectives was to analyze the impact of HMAs and LEN on the outcome of patients for whom ESA treatment failed; therefore, we considered patients having received other treatments or RBC transfusions only as the MISC group. To assess whether patients having received active treatments such as antithymocyte globulin (ATG; which might affect OS) ${ }^{13}$ could have influenced the final results of our multivariable analysis, we performed a sensitivity analysis excluding these patients.

OS was analyzed using a Cox model including these predefined covariates, considering second-line treatment as a time-dependent covariate, and AML cumulative incidence was analyzed using a Fine and Gray model, considering death as a competing risk. Progression to higher-risk MDS, considered a weaker variable than the well-documented AML transformation, was not analyzed.

The significance of the included covariates was assessed using the Wald test, proportional hazards assumptions using Schoenfeld residual analysis, and time-to-event graphical representations using the 
Kaplan-Meier method. The time-dependent impact of second-line treatments was analyzed using the method described by Simon and Makuch. ${ }^{14,15}$ Hazard ratios (HRs) and 95\% CIs were estimated, with $P$ values $<.05$ denoting significance. Statistical analyses were performed using STATA software (version 13.1; STATA, College Station, TX).

\section{RESULTS}

\section{Baseline Characteristics of Patients Experiencing Primary or Secondary Failure}

Of the 1,698 included patients, 653 (34\%) experienced primary ESA resistance and 1,045 (61.5\%) responded to ESAs, of whom 551 (32.5\%) had continued response at the time of analysis and $494(29 \%)$ had relapsed after a median response duration of 17 months (range, 3 to 74 months; Appendix Fig A1, online only). For the purpose of this study, only the 1,147 patients experiencing primary or secondary failure after ESAs were analyzed. After reclassification using IPSS-R criteria, 157 (14\%), 644 (56\%), 187 (16\%), and 159 patients (14\%), respectively, had very low, low, intermediate, and not evaluable IPSS-R scores at ESA initiation (in patients with a not evaluable score, marrow blasts were $\leq 5 \%$ ).

Baseline characteristics of the three groups (primary and secondary failure and continued responders to ESAs) differed significantly in parameters known to be associated with ESA response, including serum EPO level, IPSS-R score, and serum ferritin levels (Appendix Table A1, online only). In addition, primary resistant patients were younger, were more often male, had a lower hemoglobin level at ESA initiation, and were more likely to have RA with excess blasts or MDS with ring sideroblasts.

\section{Outcome of Patients Experiencing Primary Versus Secondary Failure}

Five-year cumulative incidence of AML transformation in patients experiencing primary or secondary failure was $13.4 \%$ ( $95 \% \mathrm{CI}, 10.9 \%$ to $16.4 \%)$; it was $8.1 \%$ (95\% CI, $5.1 \%$ to $12.8 \%$ ) in those experiencing secondary failure versus $16.7 \%$ (95\% CI, $13.4 \%$ to $20.8 \%)$ in those experiencing primary failure $(P=.001$; Appendix Fig A2, online only).

At last follow-up, 622 patients were alive and 525 had died. With a median follow-up of 41 months, median survival from ESA failure was 52.2 and 60.4 months for those with primary refractory disease and those experiencing relapse, respectively $(P=.12$; Fig 1$)$.

\section{Subsequent Lines of Treatment}

Of the 1,147 patients experiencing primary or secondary ESA failure, $450(39 \%)$ received a second-line treatment other than RBC transfusions (Appendix Table A2, online only; Appendix Fig A1), such as HMAs ( $\mathrm{n}=194$ [17\%], including 11 combined with $\mathrm{EPO})$ or LEN $(\mathrm{n}=148[13 \%]$, alone in 85 patients and combined with EPO in 63 patients, with 31 patients having received LEN $5 \mathrm{mg} /$ day and 117 LEN $10 \mathrm{mg} /$ day $).$ Other treatments $(\mathrm{n}=108)$ included thalidomide $(\mathrm{n}=19)$, all-trans-retinoic acid $(\mathrm{n}=10)$, valproic acid $(n=2)$, arsenic $(n=2)$, hydroxyurea $(n=7)$, activin soluble receptors (ligand traps; $n=2)$, low-dose cytarabine $(n=6)$, and ATG with or without ciclosporine $(\mathrm{n}=15)$. Median time from ESA failure to initiation of HMAs and LEN was 15 and 11 months,

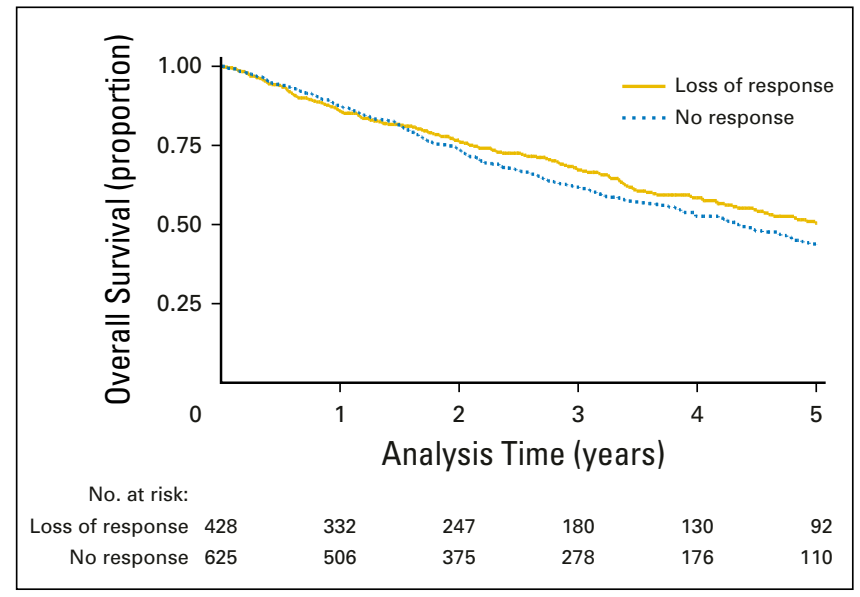

Fig 1. Kaplan-Meier estimates of overall survival from erythropoiesis-stimulating agent (ESA) failure according to primary or secondary failure after ESA treatment $(P=.12)$.

respectively $(P=.1)$. Median number of treatment cycles of HMAs and LEN received was seven and four, respectively. Response rate to HMAs and LEN (including HI-E, complete response, and partial response) was $25 \%$ and $38 \%$, respectively.

After second-line treatment, 253 patients received third-line treatment (HMAs, $\mathrm{n}=60$; LEN, $\mathrm{n}=139$; and MISC, $\mathrm{n}=54$ ), with a median interval between second- and third-line treatments of 3.6 months; 61 patients received fourth-line treatment (HMAs, $\mathrm{n}=26$; LEN, $\mathrm{n}=8$; HMAs plus LEN, $\mathrm{n}=1$; and other treatments, $\mathrm{n}=26$ ), and 14 patients received fifth-line treatment (HMAs, $\mathrm{n}=8$; LEN, $\mathrm{n}=1$; and other treatments, $\mathrm{n}=5$; Appendix Table A2). Median duration of third- and fourth-line treatments was 4.7 and 3.6 months, respectively. Twenty-eight patients received an HMA followed by LEN, and 56 patients received LEN followed by an HMA. Only two patients underwent allogeneic transplantation as third- and fifth-line treatments, respectively.

Focusing on second-line treatment to study the impact of treatment strategies after ESA failure, the baseline characteristics of patients in the MISC group versus those receiving HMAs versus LEN differed significantly in terms of age and WHO classification. Median age was higher in the MISC group (75 $v 72$ and 72 years; $P<.01)$; there were more males in the HMA group $(72 \% \mathrm{v} 61 \%$ for MISC and $62 \%$ for LEN; $P=.02$ ), more MDS with ring sideroblasts in patients treated with LEN or HMAs (65\% and 48\% v 33\%, respectively; $P<.001$ ), and more patients with an intermediate IPSS-R score at loss of ESA response among those treated with LEN $(34 \%)$ or HMAs $(33 \%)$ versus MISC $(16 \% ; P<.001$; Table 1$)$. IPSS-R score at second-line treatment initiation was available in 317 patients and was intermediate in $48 \%$ and $22 \%$ of patients receiving LEN and HMAs, respectively, compared with $23 \%$ for the MISC group.

After ESA failure, significantly more progressions to higherrisk IPSS-R groups were seen in the HMA group compared with the other treatments ( $8 \%$ for HMA $v 4 \%$ for LEN and $2 \%$ for MISC; $P<.001$ ), despite the exclusion of patients having experienced progression clearly to an intermediate-II or high IPSS score or IPSS-R high or very high score at ESA failure (Table 1). More IPSS-R intermediate risk ( $v$ good and very good risk) karyotypes 


\begin{tabular}{|c|c|c|c|c|}
\hline \multirow[b]{2}{*}{ Characteristic } & \multicolumn{3}{|c|}{ No. (\%) } & \multirow[b]{2}{*}{$P$} \\
\hline & $\begin{array}{c}\text { LEN } \\
(\mathrm{n}=148)\end{array}$ & $\begin{array}{c}\text { HMAs } \\
(n=194)\end{array}$ & $\begin{array}{c}\text { MISC } \\
(n=805)\end{array}$ & \\
\hline Age, years & & & & .01 \\
\hline Median & 72 & 72 & 75 & \\
\hline Q1-Q3 & $64-76$ & $65-78$ & $68-81$ & \\
\hline Female sex & $57(38)$ & $55(28)$ & 314 (39) & .02 \\
\hline WHO classification at ESA failure & & & & $<.001$ \\
\hline RA & $18(15)$ & $32(29)$ & $88(38)^{*}$ & \\
\hline MDS with ringed sideroblasts & $75(65)$ & $53(48)$ & $63(33)^{*}$ & \\
\hline RAEB-1 & $18(16)$ & $18(16)$ & $17(12)^{*}$ & \\
\hline MDS-U & $4(3)$ & $4(3)$ & $8(5)^{*}$ & \\
\hline IPSS-R at ESA failure & & & & $<.001$ \\
\hline Very low & $4(3)$ & $7(4)$ & $119(17)^{*}$ & \\
\hline Low & $92(63)$ & $109(63)$ & $471(67)^{*}$ & \\
\hline Intermediate & 49 (34) & $57(33)$ & $111(16)^{*}$ & \\
\hline IPSS-R progression at ESA failure & $6(4)$ & $16(8)$ & $16(2)$ & $<.001$ \\
\hline
\end{tabular}

characterized the HMA and LEN groups compared with the MISC group $(P<.001)$.

\section{Impact of Treatment Strategies After ESA Failure}

Five-year AML transformation cumulative incidence was $20.3 \%, 20.3 \%$, and $11.3 \%$ for those receiving HMAs, LEN, and MISC, respectively $(P=.05$; Fig 2$)$. In multivariable analysis, after adjusting for second-line treatment received after ESA failure as a time-dependent variable, age older than 75 years ( $\mathrm{HR}, 0.56$; $95 \% \mathrm{CI}, 0.35$ to $0.88 ; P=.01$ ) and secondary failure (with duration of response to ESA > 6 months); HR, 0.425; 95\% CI, 0.24 to 0.75 ; $P=.003)$ were associated with lower AML progression rates (Table 2). Increased AML incidence was associated with primary

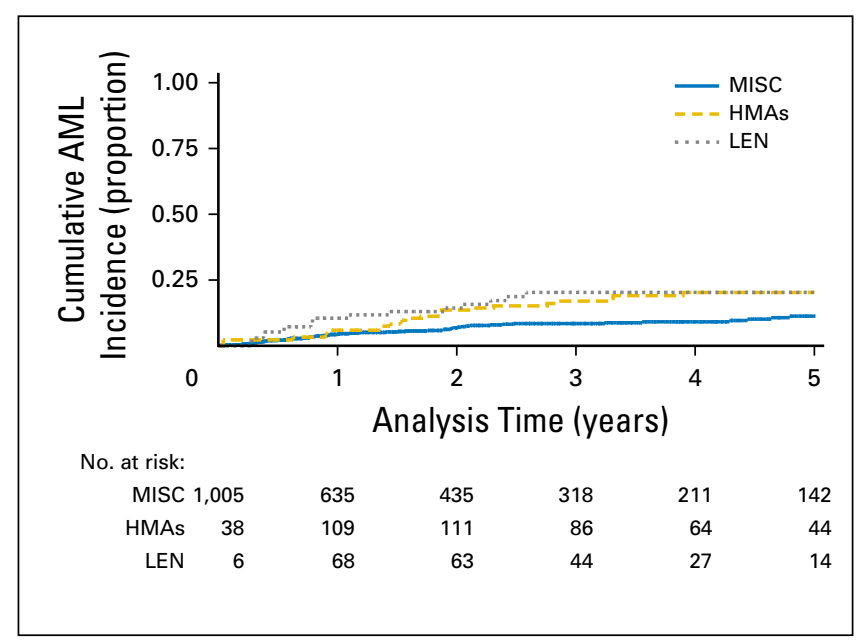

Fig 2. Simon-Makuch model (with treatment as a time-dependent variable) of cumulative acute myeloid leukemia $(A M L)$ incidence in patients receiving lenalidomide (LEN) or hypomethylating agents (HMAs) versus other treatments or RBC transfusion only (MISC) as second-line treatment (from erythropoiesis-stimulating agent failure; $P=.05$ ). versus secondary failure of ESAs rather than with IPSS-R risk at ESA failure. The HMA group had a higher risk of AML progression (HR, 1.78; $P=.02$ ), possibly related to the higher proportion of patients with an intermediate IPSS-R score at ESA failure (HR, $1.62 ; P=.19$ for IPSS-R intermediate score; Table 2).

Five-year OS after ESA failure was $36.5 \%, 41.7 \%$, and $51 \%$ after HMAs, LEN, and MISC, respectively $(P=.21$; Fig 3$)$. In a model integrating type of treatment, age, sex, and IPSS-R score at ESA failure (Table 3), age older than 75 years ( $\mathrm{HR}, 1.73 ; 95 \% \mathrm{CI}$, 1.41 to $2.13 ; P<.001)$ and intermediate IPSS-R score (HR, 2.86; 95\% CI, 1.91 to $4.30 ; P<.001)$ remained significantly associated with worse OS. There was no significant influence of treatment as a time-dependent variable on OS (HR, 1.26; 95\% CI, 0.97 to 1.64; $P=.08$ for HMA; HR, $1.13 ; 95 \%$ CI, 0.80 to $1.59 ; P=.51$ for LEN; Table 3).

In the heterogeneous MISC group, we conducted a sensitivity analysis by repeating our multivariable analyses after exclusion of patients who received other active treatments, with results (comparing patients who received HMAs $v$ LEN $v$ RBCs) similar to those obtained with the whole MISC group (Appendix Table A3, online only). Although there was no influence of treatment as a time-dependent variable on OS (HR, 1.22; 95\% CI, 0.93 to $1.58 ; P=.15$ for HMAs; HR, $1.09 ; 95 \% \mathrm{CI}, 0.77$ to 1.55; $P=.62$ for LEN), HMA use did affect risk of AML transformation (HR, 1.85; 95\% CI, 1.11 to $3.10 ; P=.02$; Table 2$)$. In the 15 patients who received ATG, 5-year OS was $50.0 \%$ and did not significantly differ from that of other patients in the MISC group.

Causes of death were predominantly infection (26\%) and AML transformation (23\%) in the HMA group and infection $(22 \%)$ and AML transformation (15\%) in the LEN group. There were no excess deaths resulting from secondary cancers in the LEN group $(2 \%)$. Deaths in the MISC group resulted from infection (20\%), AML transformation (12\%), and unknown causes (49\%), probably related to patient comorbidities. 


\begin{tabular}{|c|c|c|c|}
\hline Factor & $\mathrm{HR}$ & $95 \% \mathrm{Cl}$ & $\mathrm{P}$ \\
\hline \multicolumn{4}{|l|}{ Second-line treatment after ESAs* } \\
\hline HMAs & 1.78 & 1.08 to 2.93 & .02 \\
\hline LEN & 1.46 & 0.77 to 2.78 & .24 \\
\hline Age $>75$ years & 0.57 & 0.35 to 0.91 & .02 \\
\hline Female sext & 1.01 & 0.65 to 1.57 & .95 \\
\hline IPSS-R progression at ESA failure $\ddagger$ & 1.33 & 0.61 to 2.91 & .47 \\
\hline \multicolumn{4}{|c|}{ IPSS-R score at loss of response to ESAs $\neq$} \\
\hline Intermediate & 1.62 & 0.78 to 3.37 & .19 \\
\hline Low & 0.71 & 0.36 to 1.44 & .34 \\
\hline \multicolumn{4}{|c|}{ Secondary ESA failure, duration of response to ESAs§ } \\
\hline$\leq 6$ months & 0.67 & 0.23 to 1.93 & .46 \\
\hline$>6$ months & 0.41 & 0.23 to 0.75 & .002 \\
\hline \multicolumn{4}{|c|}{$\begin{array}{l}\text { NOTE. IPSS-R progression at ESA failure versus initiation of ESAs indicates patients whose IPSS-R score progressed between ESA failure and ESA initiation. } \\
\text { Abbreviations: AML, acute myeloid leukemia; ESA, erythropoiesis-stimulating agent; HMA, hypomethylating agent; HR, hazard ratio; IPSS-R, revised International } \\
\text { Prognostic Scoring System; LEN, lenalidomide. } \\
\text { *Other treatments or RBC transfusion only as reference group. } \\
\text { †Male sex as reference group. } \\
\text { ‡Very low risk as reference group. } \\
\text { §Primary failure as reference group. }\end{array}$} \\
\hline
\end{tabular}

\section{DISCUSSION}

This study, combining patients from eight data sets (six large retrospective registries and two prospective trials) is, to our knowledge, the largest to present the outcome of patients with lower-risk MDS who did not respond to ESAs or experienced relapse after an initial response to ESAs. We found that patients experiencing primary ESA failure had a higher risk of progression to AML than those experiencing secondary failure, although this did not translate into a significant OS difference between the two groups. Second-line treatments (HMAs and LEN) had no significant influence on OS compared with MISC.

In our study, the response rate to ESAs was $61.5 \%$, and median response duration was 17 months, similar to other

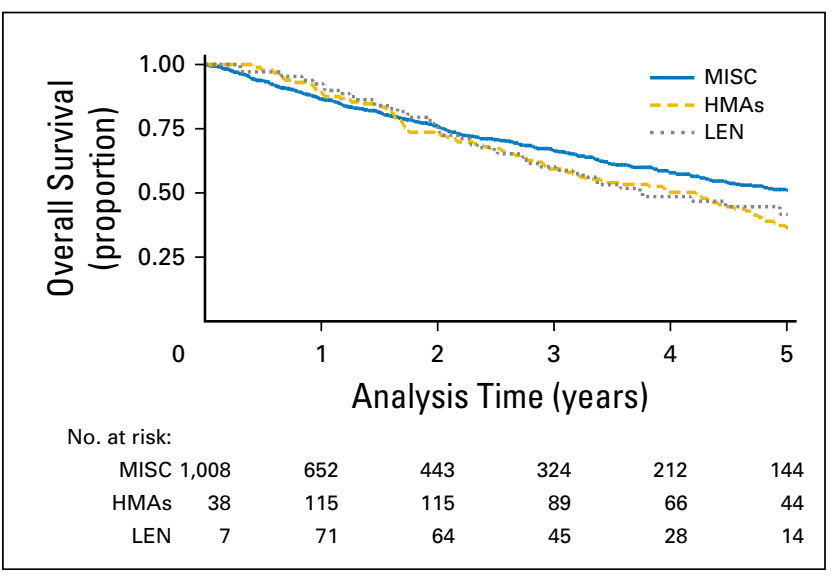

Fig 3. Simon-Makuch model (with treatment as a time-dependent variable) of overall survival in patients receiving lenalidomide (LEN) or hypomethylating agents (HMAs) at second-line treatment versus other treatments or RBC transfusion only (MISC; from erythropoiesis-stimulating agent failure; $P=0.21$ ). published results in lower-risk MDS, in which most patients have serum EPO levels < $500 \mathrm{U} / \mathrm{l}$ (in our study, median serum EPO level was $203 \mathrm{U} / \mathrm{l}$ ) and no or limited RBC transfusion requirements. In addition to well-known factors predictive of response to ESAs, including lower serum EPO level, IPSS-R score, and ferritin level, we found that MDS with ring sideroblasts were more frequent among primary refractory patients, a result described by other groups. $^{16,17}$

Median OS from ESA failure was 52 and 60 months in patients experiencing primary failure and relapse, respectively. Early ESA failure (primary failure or relapse within 6 months of response) was associated with a higher risk of AML progression, which did not translate into worse OS.

Results of our multivariable model showed that simple clinical and biologic characteristics, including age and IPSS-R score, could predict OS. This further stresses the prognostic value of IPSS-R both for responsiveness to ESA treatment ${ }^{5}$ and for outcome after ESA failure.

The second main conclusion of our work is that the introduction of an active treatment like HMAs or LEN after ESA failure had no obvious influence on OS. Main causes of death were AML transformation and infection, as previously described, which differed in the MISC group. ${ }^{18}$ The HMA group had a higher incidence of AML progression, possibly related to the higher proportion of patients with an intermediate IPSS-R score at the time of ESA failure, despite adjustment for this variable in multivariable analyses. One limitation of this study is the confounding influence of the indication for subsequent therapies in patients who may have fewer comorbidities and are thus predicted to live longer than those receiving RBC transfusions only. Despite this potential bias, no survival advantage was observed with HMA or LEN use in this selected population of lower-risk patients at ESA initiation.

Of note, our outcome analysis was performed focusing on second-line treatment, whereas some patients received several successive treatments after ESA failure. In contrast, after 


\begin{tabular}{|c|c|c|c|}
\hline Factor & $\mathrm{HR}$ & $95 \% \mathrm{Cl}$ & $P$ \\
\hline \multicolumn{4}{|l|}{ Second-line treatment after ESAs* } \\
\hline HMAs & 1.26 & 0.97 to 1.64 & .084 \\
\hline LEN & 1.12 & 0.80 to 1.59 & .51 \\
\hline Age $>75$ years & 1.74 & 1.41 to 2.12 & $<.001$ \\
\hline Female sext & 0.88 & 0.71 to 1.10 & .26 \\
\hline IPSS-R progression at ESA failure & 0.77 & 0.46 to 1.29 & .32 \\
\hline \multicolumn{4}{|l|}{ IPSS-R score at ESA failure $\neq$} \\
\hline Intermediate & 2.87 & 1.91 to 4.30 & $<.001$ \\
\hline Low & 1.33 & 0.92 to 1.93 & .12 \\
\hline \multicolumn{4}{|c|}{ Secondary ESA failure, duration of response to ESAs§ } \\
\hline$\leq 6$ months & 0.93 & 0.62 to 1.41 & .74 \\
\hline$>6$ months & 0.83 & 0.66 to 1.04 & .10 \\
\hline
\end{tabular}

Abbreviations: ESA, erythropoiesis-stimulating agent; HMA, hypomethylating agent; HR, hazard ratio; IPSS-R, revised International Prognostic Scoring System; LEN, lenalidomide; OS, overall survival.

* Other treatments or RBC transfusion only as reference group.

†Male sex as reference group.

¥Very low risk as reference group.

§Primary failure as reference group.

second-line treatment, the influence of other lines of treatment on OS could not be assessed in multivariable analyses, because of the limited number of patients in each subgroup.

In conclusion, early failure of ESAs was associated with a higher incidence of AML transformation, which did not translate into worse OS, within the limited follow-up in this study. IPSS-R intermediate score was associated with worse outcome, and primary resistance to ESAs was associated with a higher incidence of AML transformation. Second-line treatment with LEN or HMAs after ESA failure did not significantly influence OS. This study may be important in designing future clinical trials involving patients with lower-risk MDS for whom ESA treatment fails, in whom novel treatments are required. Parameters predicting worse outcome, including IPSS-R subtype and duration of response to ESAs, could indicate a more intensive therapeutic approach, including allogeneic stem-cell transplantation.

\section{AUTHORS' DISCLOSURES OF POTENTIAL CONFLICTS OF INTEREST}

Disclosures provided by the authors are available with this article at jco.org.

\section{AUTHOR CONTRIBUTIONS}

Conception and design: Sophie Park, Jean-François Hamel, Andrea Toma, Charikleia Kelaidi, Sylvain Thépot, François Dreyfus, Pierre Fenaux Provision of study materials or patients: Sophie Park, Andrea Toma, Charikleia Kelaidi, Sylvain Thépot, Maria Diez Campelo, Valeria Santini, Mikkael A. Sekeres, Enrico Balleari, Jennifer Kaivers, Rosa Sapena,

Katharina Götze, Catharina Müller-Thomas, Odile Beyne-Rauzy, Aspasia Stamatoullas, Ioannis Kotsianidis, Rami Komrokji, David P. Steensma, Jaime Fensterl, Gail J. Roboz, Teresa Bernal, Fernando Ramos, Marisa Calabuig, Agnès Guerci-Bresler, Dominique Bordessoule, Pascale ConyMakhoul, Stéphane Cheze, Eric Wattel, Christian Rose, Norbert Vey, Daniela Gioia, Dario Ferrero, Gianluca Gaidano, Giovanni Cametti, Fabrizio Pane, Alessandro Sanna, Ulrich Germing, Guillermo F. Sanz, François Dreyfus, and Pierre Fenaux

Collection and assembly of data: Sophie Park, Andrea Toma, Charikleia Kelaidi, Sylvain Thépot, Maria Diez Campelo, Valeria Santini, Mikkael A. Sekeres, Katharina Götze, Ulrich Germing, Guillermo F. Sanz

Data analysis and interpretation: Sophie Park, Jean-François Hamel, Andrea Toma, Charikleia Kelaidi, Sylvain Thépot, Maria Diez Campelo, Valeria Santini, Mikkael A. Sekeres, Enrico Balleari, Jaime Fernsterl, Guillermo F. Sanz, François Dreyfus, Pierre Fenaux

Manuscript writing: All authors

Final approval of manuscript: All authors

Accountable for all aspects of the work: All authors

\section{REFERENCES}

1. Greenberg P, Cox C, LeBeau MM, et al: International scoring system for evaluating prognosis in myelodysplastic syndromes. Blood 89:2079-2088, 1997

2. Park S, Grabar S, Kelaidi C, et al: Predictive factors of response and survival in myelodysplastic syndrome treated with erythropoietin and G-CSF: The GFM experience. Blood 111:574-582, 2008

3. Jädersten $M$, Malcovati $L$, Dybedal I, et al: Erythropoietin and granulocyte-colony stimulating factor treatment associated with improved survival in myelodysplastic syndrome. J Clin Oncol 26: 3607-3613, 2008
4. Greenberg PL, Sun Z, Miller KB, et al: Treatment of myelodysplastic syndrome patients with erythropoietin with or without granulocyte colonystimulating factor: Results of a prospective randomized phase 3 trial by the Eastern Cooperative Oncology Group (E1996). Blood 114:2393-2400, 2009

5. Santini V, Schemenau J, Levis A, et al: Can the revised IPSS predict response to erythropoieticstimulating agents in patients with classical IPSS low or intermediate-1 MDS? Blood 122:2286-2288, 2013

6. Kelaidi C, Beyne-Rauzy O, Braun T, et al: High response rate and improved exercise capacity and quality of life with a new regimen of darbepoetin alfa with or without filgrastim in lower-risk myelodysplastic syndromes: A phase II study by the GFM. Ann
Hematol 92:621-631, 2013 [Ann Hematol: 92:633, 2013]

7. Kelaidi C, Park S, Sapena R, et al: Long-term outcome of anemic lower-risk myelodysplastic syndromes without $5 q$ deletion refractory to or relapsing after erythropoiesis-stimulating agents. Leukemia 27:1283-1290, 2013

8. Toma A, Kosmider O, Chevret $\mathrm{S}$, et al: Lenalidomide with or without erythropoietin in transfusiondependent erythropoiesis-stimulating agent-refractory lower-risk MDS without $5 q$ deletion. Leukemia 30: 897-905, 2016

9. Thépot $S$, Ben Abdelali $R$, Chevret $S$, et al: A randomized phase II trial of azacitidine $+/$ - epoetin- $\beta$ in lower-risk myelodysplastic syndromes resistant to erythropoietic stimulating agents. Haematologica 101:918-925, 2016 
10. Henderson V, Velleman PF: Building multiple regression models interactively. Biometrics 37 : 391-411, 1981

11. Derksen S, Keselman HJ: Backward, forward and stepwise automated subset selection algorithms: Frequency of obtaining authentic and noise variables. Br J Math Stat Psychol 45:265-282, 1992

12. Hurvich $\mathrm{CM}$, Tsai $\mathrm{CL}$ : The impact of model selection on inference in linear regression. Am Stat 44:214-217, 1990

13. Sloand EM, Wu CO, Greenberg $P$, et al: Factors affecting response and survival in patients with myelodysplasia treated with immunosuppressive therapy. J Clin Oncol 26:2505-2511, 2008

14. Makuch $R$, Simon R: Recommendations for the analysis of the effect of treatment on the development of second malignancies. Cancer 44: 250-253, 1979

15. Schultz LR, Peterson EL, Breslau N: Graphing survival curve estimates for time-dependent covariates. Int J Methods Psychiatr Res 11:68-74, 2002

16. Hellström-Lindberg $E$, Negrin $R$, Stein $R$, et al: Erythroid response to treatment with G-CSF plus erythropoietin for the anaemia of patients with myelodysplastic syndromes: Proposal for a predictive model. Br J Haematol 99:344-351, 1997

17. Tehranchi $R$, Fadeel $B$, Forsblom AM, et al: Granulocyte colony-stimulating factor inhibits spontaneous cytochrome c release and mitochondriadependent apoptosis of myelodysplastic syndrome hematopoietic progenitors. Blood 101:1080-1086, 2003

18. Nachtkamp K, Stark R, Strupp $C$, et al: Causes of death in 2877 patients with myelodysplastic syndromes. Ann Hematol 95:937-944, 2016

\section{Affiliations}

Sophie Park, Centre Hospitalier Universitaire (CHU) Grenoble Alpes, Université Grenoble Alpes, Grenoble; Jean-François Hamel and Sylvain Thépot, CHU d'Angers; Sylvain Thépot, Université d'Angers, Institut National de la Santé et de la Recherche Médicale, Unité 1232, and LabEx IGO, Angers; Andrea Toma, CHU Henri Mondor, Assistance Publique Hôpitaux de Paris (AP-HP), Université Paris-Est Créteil; Rosa Sapena and Pierre Fenaux, CHU Saint Louis, AP-HP, Université Paris X; François Dreyfus, CHU Cochin, AP-HP, Université Paris V, Paris; Charikleia Kelaidi, CHU Avicenne, Avicenne; Odile Beyne-Rauzy, Institut Universitaire du Cancer de Toulouse Oncopole, Toulouse; Aspasia Stamatoullas, Centre Henri Becquerel, Rouen; Agnès Guerci-Bresler, CHU Nancy, Nancy; Dominique Bordessoule, CHU Dupuytren, Limoges; Pascale Cony-Makhoul, Centre Hospitalier Annecy Genevois, Epagny Metz-Tessy; Stéphane Cheze, CHU de Caen, Caen; Eric Wattel, Centre Hospitalier Lyon-Sud, Pierre Bénite, Lyon; Christian Rose, Université Catholique de Lille, Lille; Norbert Vey, Institut Paoli-Calmettes Marseille, Marseille, France; Maria Diez Campelo, Hospital Universitario de Salamanca, Salamanca; Teresa Bernal, Hospital Central de Asturias, Oviedo; Fernando Ramos, Hospital de León, León; Marisa Calabuig, Hospital Clínico de Valencia; Guillermo F. Sanz, Hospital Universitario y Politecnico La Fe, Valencia, Spain; Valeria Santini and Alessandro Sanna, Azienda Ospedaliero Universitaria (AOU) Careggi and University of Florence, Florence; Enrico Balleari, Istituto di Ricovero e Cura a Carattere Scientifico AOU San Martino di Genova, Genova; Daniela Gioia, Azienda Ospedaliera SS Antonio e Biagio e Cesare Arrigo, Alessandria; Dario Ferrero, Università degli Studi di Torino; Gianluca Gaidano, Amedeo Avogadro University of Eastern Piedmont, Torino; Giovanni Cametti, Ospedale Maggiore di Chieri, Chieri; Fabrizio Pane, Università Federico II, Napoli, Italy; Mikkael A. Sekeres and Jaime Fensterl, Cleveland Clinic Taussig Cancer Institute, Cleveland, OH; Rami Komrokji, H. Lee Moffitt Cancer Center and Research Institute, Tampa, FL; David P. Steensma, Dana-Farber Cancer Institute, Boston, MA; Gail J. Roboz, Weill Medical College of Cornell University, New York, NY; Jennifer Kaivers and Ulrich Germing, University of Düsseldorf, Düsseldorf; Katharina Götze and Catharina Müller-Thomas, Universität München, München, Germany; and Ioannis Kotsianidis, Democritus University of Thrace, University Hospital of Alexandroupolis, Alexandroupolis, Greece.

\section{Support}

Supported in part through the Clinical Research Consortium by the Edward P. Evans Foundation.

\section{Prior Presentation}

Presented in part at the 13th International Symposium on Myelodysplastic Syndromes, Washington, DC, April 29-May 2, 2015; the 20th Congress of the European Hematology Association, Vienna, Austria, June 11-14, 2015; and the 57th Annual Meeting of the American Society of Hematology, Orlando, FL, December 5-8, 2015. 
Outcome of Lower-Risk Patients With Myelodysplastic Syndromes Without 5q Deletion After Failure of Erythropoiesis-Stimulating Agents

The following represents disclosure information provided by authors of this manuscript. All relationships are considered compensated. Relationships are self-held unless noted. I = Immediate Family Member, Inst = My Institution. Relationships may not relate to the subject matter of this manuscript. For more information about ASCO's conflict of interest policy, please refer to www.asco.org/rwc or ascopubs.org/jco/site/ifc.

Sophie Park

Consulting or Advisory Role: Novartis

Research Funding: Novartis, Celgene

Jean-François Hamel

No relationship to disclose

Andrea Toma

No relationship to disclose

Charikleia Kelaidi

No relationship to disclose

Sylvain Thépot

Honoraria: Celgene

Travel, Accommodations, Expenses: Jazz Pharmaceuticals

Maria Diez Campelo

Honoraria: Celgene, Novartis

Consulting or Advisory Role: Celgene, Novartis

Speakers' Bureau: Celgene, Novartis

Research Funding: Janssen Pharmaceuticals, Celgene, Novartis

Valeria Santini

Honoraria: Novartis, Janssen-Ortho, Astex Pharmaceuticals, Amgen

Research Funding: Celgene

Mikkael A. Sekeres

Consulting or Advisory Role: Celgene, Millennium Pharmaceuticals

Enrico Balleari

No relationship to disclose

Jennifer Kaivers

No relationship to disclose

Rosa Sapena

No relationship to disclose

Katharina Götze

Honoraria: Celgene, Novartis, Janssen Pharmaceuticals, Bristol-Myers Squibb

Catharina Müller-Thomas

No relationship to disclose

Odile Beyne-Rauzy

Research Funding: Novartis (Inst)

Aspasia Stamatoullas

Honoraria: Celgene

Ioannis Kotsianidis

No relationship to disclose

Rami Komrokji

Stock or Other Ownership: AbbVie

Consulting or Advisory Role: Incyte, Celgene, Novartis

Speakers' Bureau: Novartis, Alexion Pharmaceuticals

Research Funding: Incyte (Inst), Celgene (Inst), GlaxoSmithKline (Inst), Eleos (Inst)

Travel, Accommodations, Expenses: Celgene, Incyte, Alexion

Pharmaceuticals, Novartis
David P. Steensma

Consulting or Advisory Role: Celgene, Akebia, Takeda Pharmaceuticals, H3, Pfizer, Janssen-Cilag

Jaime Fernsterl

No relationship to disclose

Gail J. Roboz

Consulting or Advisory Role: Agios, Amphivena, AstraZeneca, Boehringer Ingelheim, GlaxoSmithKline, Janssen Pharmaceuticals, MEI Pharma, Roche, Shire, Amgen, Astex Pharmaceuticals, Celator, Celgene, Cellectis, CTI BioPharma, Genoptix, Juno Therapeutics, MedImmune, Novartis, Onconova Therapeutics, Pfizer, Sunesis Pharmaceuticals Research Funding: AbbVie (Inst), Agios (Inst), Astex Pharmaceuticals (Inst), Celgene (Inst), CTI BioPharma (Inst), Karyopharm Therapeutics (Inst), MedImmune (Inst), MEI Pharma (Inst), Moffitt (Inst), Novartis (Inst), Onconova Therapeutics (Inst), Pfizer (Inst), Sunesis Pharmaceuticals (Inst), Tensha Therapeutics (Inst), Cellectis (Inst) Travel, Accommodations, Expenses: AstraZeneca, Shire, Astellas Pharma, Celator, Incyte, Roche, Amphivena, MEI Pharma, Astex Pharmaceuticals, Janssen Pharmaceuticals, Juno Therapeutics

\section{Teresa Bernal}

Travel, Accommodations, Expenses: Celgene

Fernando Ramos

Honoraria: Celgene, Janssen Pharmaceuticals, GlaxoSmithKline, Novartis, Amgen, Pfizer, Merck Sharp \& Dohme

Consulting or Advisory Role: Celgene, Janssen Pharmaceuticals, Amgen Research Funding: Celgene

Travel, Accommodations, Expenses: Celgene, Janssen Pharmaceuticals, Amgen, Roche

Marisa Calabuig

No relationship to disclose

Agnès Guerci-Bresler

Consulting or Advisory Role: Bristol-Myers Squibb, Novartis, Pfizer

Travel, Accommodations, Expenses: Bristol-Myers Squibb

Dominique Bordessoule

No relationship to disclose

Pascale Cony-Makhoul

Consulting or Advisory Role: Novartis

Speakers' Bureau: Novartis

Stéphane Cheze

Consulting or Advisory Role: Novartis

Eric Wattel

Research Funding: Novartis

Christian Rose

No relationship to disclose

Norbert Vey

Honoraria: Celgene, Novartis, Amgen, Bristol-Myers Squibb

Consulting or Advisory Role: Novartis, Roche

Travel, Accommodations, Expenses: Novartis, Amgen

Daniela Gioia

No relationship to disclose 


\section{Dario Ferrero}

Travel, Accommodations, Expenses: Novartis, Celgene, Bristol-Myers Squibb

Gianluca Gaidano

No relationship to disclose

Giovanni Cametti

No relationship to disclose

Fabrizio Pane

No relationship to disclose

Alessandro Sanna

No relationship to disclose
Ulrich Germing

Honoraria: Janssen-Cilag, Amgen, Celgene, Novartis

Guillermo F. Sanz

Honoraria: Celgene

Consulting or Advisory Role: Amgen, Janssen Pharmaceuticals

François Dreyfus

No relationship to disclose

Pierre Fenaux

No relationship to disclose 


\section{Appendix}

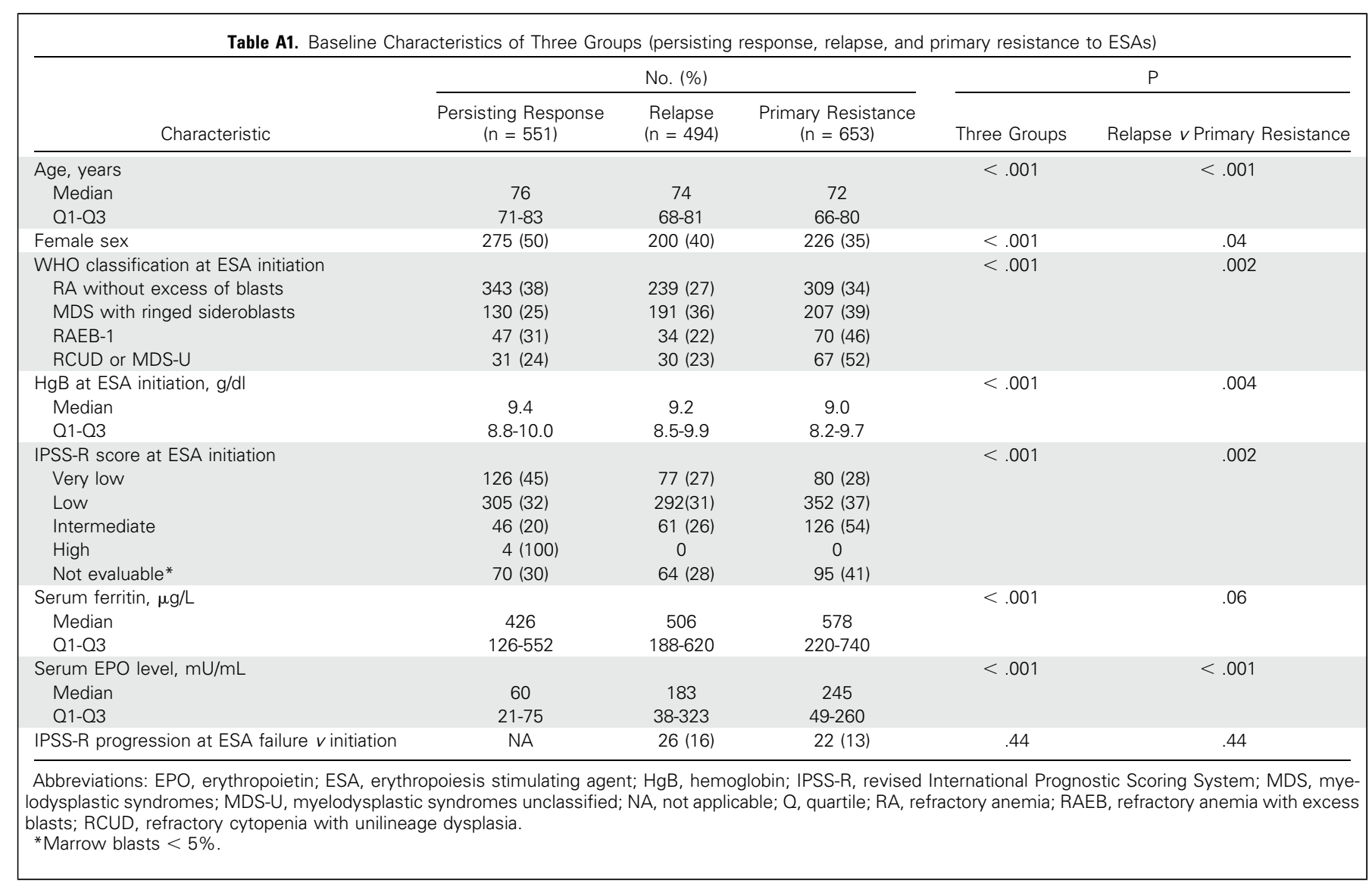

\begin{tabular}{|c|c|c|c|}
\hline \multirow[b]{2}{*}{ Treatment } & \multicolumn{3}{|c|}{ Treatment Line (No. of patients) } \\
\hline & Second & Third & Fourth \\
\hline HMAs & 194 & 60 & 26 \\
\hline LEN & 148 & 139 & 9 \\
\hline Other* & 108 & 54 & 26 \\
\hline \multicolumn{4}{|c|}{$\begin{array}{l}\text { Abbreviations: ESA, erythropoiesis stimulating agent; HMA, hypomethylating } \\
\text { agent; LEN, lenalidomide. } \\
\text { *Valproic acid, ACE-536 or }-011 \text {, thalidomide, antithymocyte globulin } \pm \text { ciclo- } \\
\text { sporine, low-dose cytarabine, hydroxyurea, or all-trans-retinoic acid. }\end{array}$} \\
\hline
\end{tabular}




\begin{tabular}{|c|c|c|c|}
\hline Variable & $\mathrm{HR}$ & $95 \% \mathrm{Cl}$ & $P+$ \\
\hline \multicolumn{4}{|l|}{ Second-line treatment $\ddagger$} \\
\hline HMAs & 1.217 & 0.934 to 1.586 & .146 \\
\hline LEN & 1.093 & 0.772 to 1.548 & .615 \\
\hline Age $>75$ years & 1.701 & 1.380 to 2.096 & $<.001$ \\
\hline \multicolumn{4}{|l|}{ gender (ref: male) } \\
\hline Female sex§ & 0.848 & 0.682 to 1.055 & .140 \\
\hline IPSS-R progression at ESA failure $v$ initiation & 0.753 & 0.449 to 1.262 & .281 \\
\hline \multicolumn{4}{|l|}{ IPSS-R score at ESA failure } \\
\hline Intermediate & 2.908 & 1.925 to 4.391 & $<.001$ \\
\hline Low & 1.353 & 0.931 to 1.967 & .113 \\
\hline Duration of response $>6$ months & 0.840 & 0.669 to 1.054 & .132 \\
\hline \multicolumn{4}{|c|}{$\begin{array}{l}\text { Abbreviations: ATG, antithymocyte globulin; ESA, erythropoiesis stimulating agent; HMA, hypomethylating agent; HR, hazard ratio; IPSS-R, revised International } \\
\text { Prognostic Scoring System; LEN, lenalidomide; MISC, other treatments or RBC transfusion only; OS, overall survival. } \\
\text { *Excluding patients who received active treatment (defined as ATG [n = 15]) in the MISC group. } \\
\text { †From z score. } \\
\text { ‡MISC without ATG as reference group. } \\
\text { §Male sex as reference group. }\end{array}$} \\
\hline
\end{tabular}

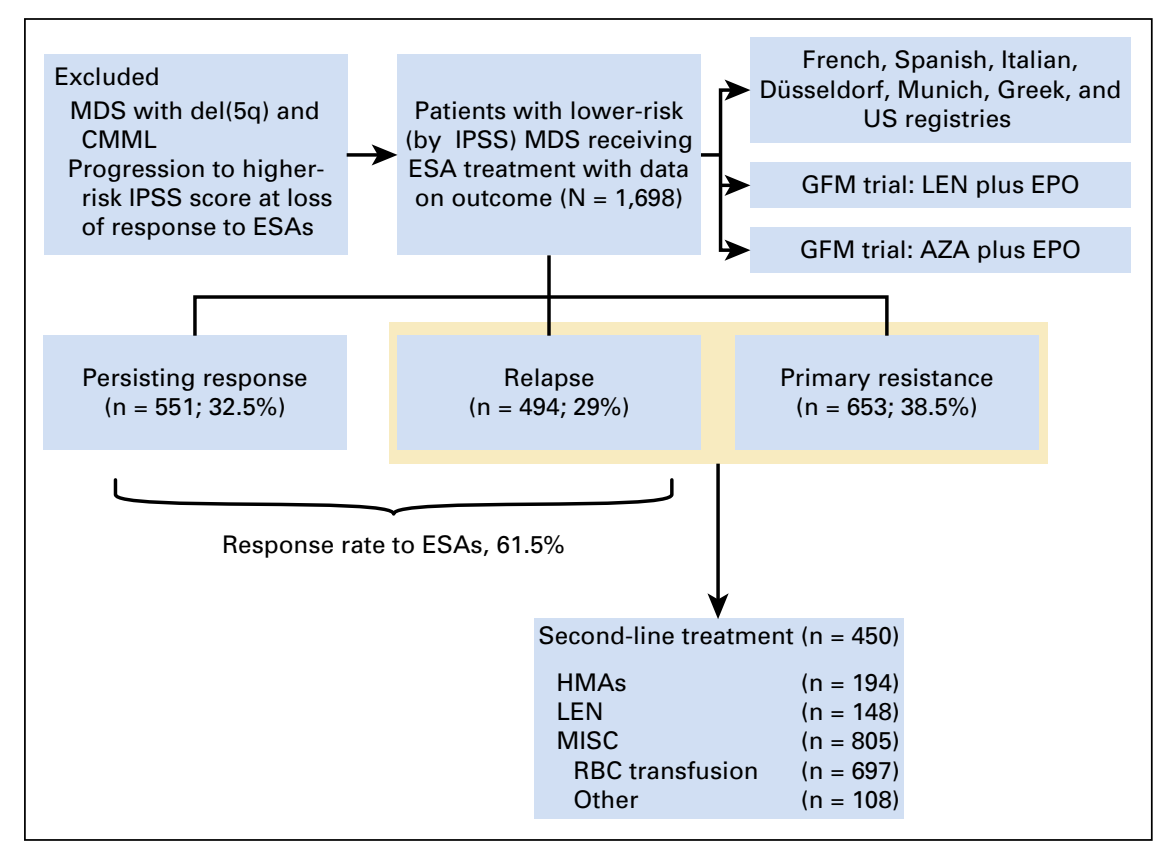

Fig A1. CONSORT diagram. AZA, azacitidine; CMML, chronic myelomonocytic leukemia; EPO, erythropoietin; ESA, erythropoiesis-stimulating agent; GFM, Groupe Francophone des Myelodysplasies; HMA, hypomethylating agent; IPSS, International Prognostic Scoring System; LEN, lenalidomide; MDS, myelodysplastic syndromes; MISC, other treatments or RBC transfusion only. 
Park et al

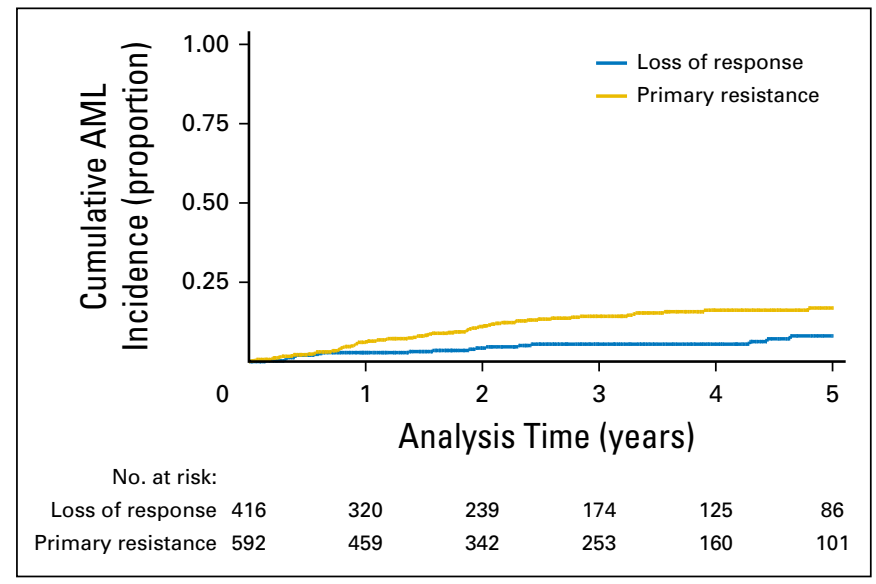

Fig A2. Cumulative acute myeloid leukemia ( $A M L)$ incidence according to primary (primary resistance) or secondary failure (loss of response; $P<.001$ ). 